Kompass

Autoimmun

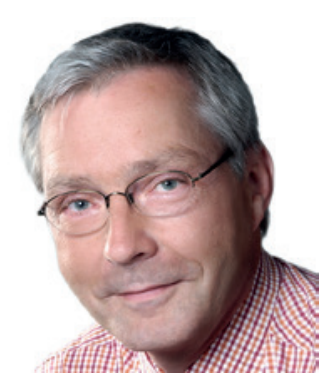

\title{
Psoriasis und Multiple Sklerose: Zusammenhänge und Risiken
}

\author{
Michael Sticherling
}

Hautklinik, Psoriasiszentrum, Universitätsklinikum Erlangen, Deutsches Zentrum für Immuntherapie, Erlangen, Deutschland

Zusammenfassung zu Silfvast-Kaiser AS, Homan KB, Mansouri B: A narrative review of psoriasis and multiple sclerosis: links and risks. Psoriasis (Auckl). 2019;9:81-90.

\section{Schlüsselwörter}

Psoriasis · Multiple Sklerose · Zusammenhang · Risikofaktor

\section{Zusammenfassung}

Bei der Psoriasis (PsO) und Multiplen Sklerose (MS) handelt es sich um zwei chronisch-entzündliche T-zellulär vermittelte Erkrankun- gen, die beide Assoziationen mit anderen inflammatorischen und autoimmunen Erkrankungen zeigen. Die Korrelation von $\mathrm{PsO}$ und MS ist allerdings aufgrund der geringen und widersprüchlichen Studienlage umstritten.

@ 2020 S. Karger GmbH, Freiburg 


\section{Transfer in die Praxis}

\section{Hintergrund}

Die Psoriasis (PsO) und Multiple Sklerose (MS) sind zwei chronischentzündliche, T-zellulär vermittelte Erkrankungen. Beide Erkrankungen zeigen Assoziationen mit anderen inflammatorischen und autoimmunen Erkrankungen. Das gemeinsame Auftreten ist klinisch und epidemiologisch bis heute kontrovers. Unter therapeutischen und prognostischen Gesichtspunkten sind jedoch solche Zusammenhänge hoch bedeutsam.

\section{Gemeinsame Zytokine bei beiden Erkrankungen}

Dazu geben Silvast-Kaiser et al. einen umfassenden und kritischen Überblick, indem sie zunächst beide Erkrankungen mit ihrem ätiopathogenetischen Hintergrund einzeln vorstellen und dann Gemeinsamkeiten herausarbeiten. Demnach ist die IL-23/IL-17-Achse bei beiden Erkrankungen bedeutsam, indem IL-23 Th17-Zellen induziert. Auch TNFa spielt eine wichtige Rolle und konnte bei beiden Erkrankungen erhöht nachgewiesen werden. Ein relativ neu entdecktes Zytokin, IL-27, das die Differenzierung von Th1-Zellen und eine Hemmung der Differenzierung von Th17-Zellen induziert, konnte sowohl bei MS als auch PsO nachgewiesen werden. Während MS-Patienten erniedrigte Spiegel von IL-27 und damit eine verminderte Th17-Inhibition zeigen, finden sich bei $\mathrm{PsO}$-Patienten höhere Spiegel, die vermutlich eine Ausweitung der Entzündung vermindern. Klinische Hinweise bestehen tatsächlich, dass bei gemeinsamem Auftreten beider Erkrankungen die MS langsamer fortschreitet, was möglicherweise durch die höhere Expression von IL-27 vermittelt wird.

Weiterhin ist klinisch beschrieben, dass sich eine MS unter therapeutischer TNF-Blockade verschlechtern kann, weshalb diese bei vorbestehender Erkrankung als relativ kontraindiziert gilt. Andererseits konnte in Studien nachgewiesen werden, dass die Wahrscheinlichkeit, bei Auftreten beider Erkrankungen eine $\mathrm{PsO}$ zu verbessern, eine Potenz höher ist als die, durch eine TNF-Blockade zu schädigen. Insgesamt bleibt damit die therapeutische TNF-Blockade bei kombinierter Erkrankung möglich, sollte jedoch kritisch abgewogen werden.

\section{Assoziation PsO und MS?}

Hinsichtlich der Korrelation von PsO und MS finden sich widersprüchliche Daten. Mindestens 6 Studien konnten ein signifikant vermehrtes Zusammentreffen beider Erkrankungen mit Inzidenzraten von 1,78 bis 4,5 pro 10000 Personenjahre nachweisen. Die $\mathrm{PsO}$ geht in vielen Fällen der MS voraus. 2 Metaanalysen konnten ein PsO-Risiko von etwa 1,3 bei MS-Patienten nachweisen. Alle diese Studien waren retrospektiv, Fall-Kontrollstudien oder fußten auf
Registerdaten, die unter nicht sicherer Verlässlichkeit der Diagnosen und inkonsequenter Meldung leiden. Mindestens 3 Studien haben keinerlei Korrelation nachgewiesen, insbesondere eine der wohl größten publizierten multizentrischen Studien, die Patienten, erstgradig Verwandte und nicht-verwandte Familienmitglieder umfasste und keinen Unterschied zwischen den Gruppen nachweisen konnte. Ein systematischer Review aus 2015 konnte eine Prävalenz der PsO bei MS-Patienten zwischen 0,4 und knapp 8\% nachweisen, eingeschlossen waren MS-Patienten, die auf PsO untersucht wurden. Auch hier sind alle Studien klein und meistens Beobachtungs- oder retrospektive Studien.

\section{Therapie von PsO und MS}

Hinsichtlich der Betreuung von MS-Patienten stehen vor allem für die schubförmige Form mehr Therapiemöglichkeiten zur Verfügung, die u.a. Fumarate, Interferon- $\beta$ und therapeutische Antikörper umfassen. Während unter Fumaraten eine Besserung der PsO zu erwarten ist, kann unter Interferontherapie eine Exazerbation auftreten. Andererseits sind in der Psoriasis-Therapie eingesetztes Methotrexat und Ciclosporin auch für die MS indiziert. Therapeutische Antikörper der PsO wie Secukinumab und Ustekinumab sowie die häufig genutzte Fototherapie werden als risikoarm angesehen. Zukünftige Entwicklungen sehen Potenzial in IL-35, das zur IL-12-Zytokin-Familie gehört und eine wichtige primäre Rolle in der Modulation von regulatorischen T-Zellen spielt, als auch in mesenchymalen Stammzellen, die in tierexperimentellen Studien positive Effekte bei $\mathrm{PsO}$ und MS gezeigt haben.

\section{Fazit für die Praxis}

Auch wenn pathogenetisch Überschneidungen bestehen, sind insgesamt die vorliegenden Daten zur Assoziation von PsO und MS widersprüchlich und nur von geringer Evidenz. Offensichtlich ist das gleichzeitige Auftreten beider Erkrankungen eher selten. Trotzdem sollten die beteiligten medizinischen Spezialitäten Dermatologie und Neurologie wechselseitig ihre Patienten kritisch begleiten und behandeln.

\section{Disclosure Statement}

Hiermit erkläre ich, dass keine Interessenskonflikte in Bezug auf den vorliegenden Kommentar bestehen.

Kontaktadresse: Prof. Dr. Michael Sticherling, Hautklinik, Universitätsklinikum Erlangen, Deutschen Zentrum für Immuntherapie, Ulmenweg 18, 91054 Erlangen, Deutschland, michael.sticherling@uk-erlangen.de 